\title{
BMJ Open Tailored digital behaviour change intervention with e-referral system to increase attendance at NHS stop smoking services (the MyWay project): study protocol for a randomised controlled feasibility trial
}

Emily Fulton, ${ }^{\oplus}$ Katie Newby, ${ }^{1}$ Kajal Gokal, ${ }^{1}$ Kayleigh Kwah, ${ }^{1}$ Lauren Schumacher, ${ }^{1}$ Louise J Jackson, ${ }^{2}$ Felix Naughton, ${ }^{3}$ Tim Coleman, ${ }^{4}$ Katherine Brown ${ }^{1}$

To cite: Fulton E, Newby K, Gokal $\mathrm{K}$, et al. Tailored digital behaviour change intervention with e-referral system to increase attendance at NHS stop smoking services (the MyWay project): study protocol for a randomised controlled feasibility trial. BMJ Open 2019;9:e028721. doi:10.1136/ bmjopen-2018-028721

- Prepublication history for this paper is available online. To view these files please visit the journal online (http://dx.doi. org/10.1136/bmjopen-2018028721).

Received 7 January 2019 Revised 29 January 2019 Accepted 30 January 2019

Check for updates

(C) Author(s) (or their employer(s)) 2019. Re-use permitted under CC BY-NC. No commercial re-use. See rights and permissions. Published by BMJ.

For numbered affiliations see end of article.

Correspondence to

Dr Emily Fulton;

ab2399@coventry.ac.uk

\section{ABSTRACT}

Introduction In the UK, smokers who use stop smoking services (SSSs) are four times more likely to stop smoking than smokers who do not. Attendance has declined, warranting the development of interventions to address this. StopApp is a novel, brief online behaviour change intervention designed to address common barriers to SSS attendance. It links to widely commissioned service management software that enables instant appointment booking at a user's location and time of choice.

Methods and analysis A two-arm parallel group, individual participant feasibility randomised controlled trial of StopApp (intervention) compared with the standard promotion of and referral to SSSs (control). The study includes a nested qualitative process evaluation to assess the acceptability of the research processes, with a subsample of participants. Smokers aged over 16 years will be recruited via three routes: General Practice (GP), community settings and online. After consenting and the collection of baseline data, participants will be randomised to control or intervention groups. Participants in the intervention group receive a link to StopApp and those in the control group receive standard web-based information about the SSSs. All participants are told they can book a SSS appointment but are under no obligation to do so. Online follow-up 2 months post randomisation includes data on SSS use and carbon monoxide verified 4-week quit rates. The study aims to recruit 162 smokers.

Ethics and dissemination Ethics approval has been granted by the West Midlands-Edgbaston NHS Research Ethics Committee. The findings will be reported in conferences and peer-reviewed publications; and will be used to design the parameters necessary for a definitive trial to ascertain the effectiveness of StopApp at increasing booking and attendance at SSSs compared with existing methods for encouraging uptake.

\section{INTRODUCTION}

Smoking remains a leading cause of mortality and morbidity worldwide. ${ }^{1}$ In addition, the direct costs of smoking to the UK National

\section{Strengths and limitations of this study}

- A feasibility randomised controlled trial with nested qualitative evaluation to ascertain acceptability of trial processes.

Both objective and subjective measures are included to assess 4-week quit rates.

- Comparison of different sources of recruitment, including GP, community settings and online social media.

- Potential for selection bias including the requirement for participants to have access to the internet.

- Reliance on self-report data only for those in the control arm.

Health Service (NHS) have been estimated at between $£ 2.7$ billion and $£ 5.2$ billion, which is equivalent to around $5 \%$ of the total NHS budget each year. ${ }^{2}$ NHS stop smoking services (SSSs) provide free and tailored support to help people stop smoking, with the use of pharmacological and behavioural interventions. The services are available to smokers over 12 years of age, including those who are pregnant. The effectiveness of SSSs is typically judged by the number of smokers who set a quit date and are abstinent from smoking 4 weeks later, verified as standard by carbon monoxide $(\mathrm{CO})$ testing. ${ }^{3}$ Four-week quit rates are a reliable predictor of long-term abstinence, with studies showing that collecting further follow-up data at 6 months provides only a modest increase in accuracy. ${ }^{4}$ Based on these measures, smokers who attend SSSs have been found to be four times more likely to quit smoking, than those who attempt to quit alone. $^{5}$ 
Although most smokers want to quit, and between $22 \%$ and $31 \%$ of them will make at least one attempt to quit each year, ${ }^{6}$ SSSs currently reach only $5 \%-10 \%$ of the smoking population. ${ }^{7}$ In addition, despite the effectiveness of SSSs, ${ }^{6}$ relative to the number of smokers, uptake has declined in recent years. ${ }^{8}$ This may be explained in part by the recent proliferation of electronic cigarettes (EC) leading people to switch to these instead of quitting smoking or instead of accessing support to quit. However, $95 \%$ of SSS practitioners have encountered clients that use EC, suggesting that smokers do not view SSS and EC use as mutually exclusive. ${ }^{9} \mathrm{~A}$ third of smokers in England have used EC at least once, ${ }^{10}$ and while their use is associated with significant reductions in numbers of cigarettes smoked this has not led to an overall rise in quit attempts. ${ }^{11}$ Therefore, SSSs are still needed to support quit attempts.

A range of studies conducted since the commissioning of SSSs began, suggest that smokers (in particular those from lower socioeconomic status [SES] groups) are often unaware that SSSs exist, or what type of service they offer. ${ }^{12-16}$ Other barriers to accessing SSSs include holding beliefs that the services lack efficacy, and will be impersonal, judgmental and not tailored to individual needs. Additionally seeking help to stop is viewed as a sign of personal weakness. ${ }^{12-14} 17$ Typically, these barriers to service uptake have not formed the focus of interventions or health promotion campaigns targeted at smokers, possibly because access to SSSs had been robust and growing until recently. ${ }^{18}$ Research has shown, however, that booklets explaining the efficacy of services ${ }^{19}$ and proactively recruiting smokers through General Practice (GP) can increase attendance at SSSs and 4-week quit rates. ${ }^{20} \mathrm{~A}$ recent trial (Start2quit) assessing personalised risk information in the form of a letter from patients' GPs and an offer of SSS taster sessions, was both effective and cost-effective at increasing SSS uptake. ${ }^{21} 22$

StopApp is a brief, novel web-based behaviour change intervention, developed with input from smokers across the SES spectrum, that targets the known barriers to service access to improve users' motivation and capability to access SSS. StopApp links to an existing online booking and service management system used by pharmacies offering SSS (PharmOutcomes), providing the opportunity to instantly book a first appointment at SSSs at a time and location to suit the user. A feasibility trial of StopApp (the MyWay study) is required to establish whether a future randomised controlled trial (RCT) can be achieved. This is especially necessary due to (1) the novelty of the StopApp intervention; (2) the potential for overlooking health inequalities associated with smoking when using digital interventions and (3) the limited extant research employing the required trial design across recruitment contexts that replicate real-world use of the intervention (including online and community settings).

\section{Aims and objectives}

The aim of the proposed research is to establish the feasibility of a future RCT of the StopApp intervention.

\section{Primary objective}

To conduct a feasibility trial of StopApp to estimate recruitment and attrition rates of participants across three settings: GP surgeries, community settings and online; at baseline, intervention access and 2-month follow-up.

\section{Secondary objectives}

Secondary objectives of the MyWay feasibility trial are to estimate the:

1. Acceptability of randomisation and the StopApp intervention for participants.

2. Acceptability of the outcome measures and measures required for cost-effectiveness analyses in a future trial.

3. Key costs that would be incurred in delivering the intervention and usual care, including a comparison of 'Did-not-attend' (DNA) rates between each arm of the trial.

4. Feasibility of accessing SSS and GP data (if recruited via GP) on attendance, quit dates set and 4-week abstinence rates for trial participants.

5. Any differential recruitment and attrition rates across socioeconomic groups, age and gender.

6. Rate of SSSs attendance in the treatment and control groups to estimate the event rate of the primary outcome measure (eg, 4-week abstinence rate) for a future trial and to support future trial sample size calculations.

\section{METHODS AND ANALYSIS}

\section{Study design}

This study is a two-arm parallel group individual participant feasibility RCT of StopApp (intervention) compared with standard promotion of and referral to SSSs (control). The study will also have a nested qualitative process evaluation to assess the acceptability of the research processes, randomisation, measures and the intervention with a subsample of participants.

Important changes to methods after pilot trial commencement Shortly after the trial commenced, it was clear that recruitment, in particular via GP practices, was slow. Observation of analytics data regarding visits to the research platform indicated that many potential participants were clicking though but not registering and taking part. In response to this, we decided to offer a financial incentive (reimbursement) to participants joining the study, for their time to complete both measures in the form of a $£ 10$ e-gift token. It was intended that this would also reduce dropout at follow-up. The changes were approved by both the funder and ethics board. Participants already recruited to the study were contacted and offered the voucher. 


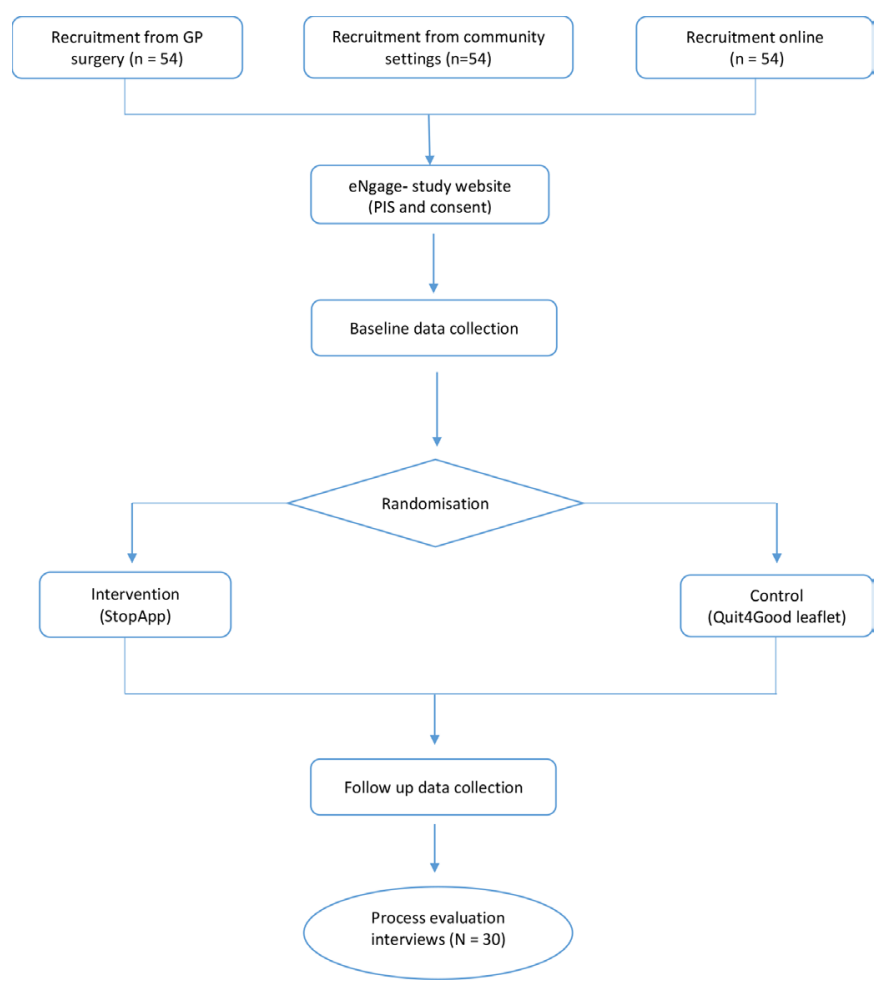

Figure 1 Flow diagram illustrating participant's route through the project.

\section{Participants}

\section{Eligibility criteria}

All current smokers aged over 16 years registered with participating GP practices in Warwickshire, or accessing participating community services or viewing advertising for the study online, will be invited to take part, regardless of whether they have previously attended SSS. Access to the internet via a computer or smartphone to complete study measures, view intervention or control content and receive SMS reminders will be a requirement.

\section{Setting}

Smokers will be recruited from three settings across Warwickshire including six GP surgeries, a range of community settings (eg, children's centres, libraries and well-being hubs) and online via social media platforms.

\section{Recruitment}

Recruitment will take place in three settings: GP practices, community settings and online.

\section{Recruitment via GP surgery}

The Clinical Research Network is supporting recruitment via GP surgeries. GP practices in Warwickshire have been invited to participate and of those who are interested, we will select six for the feasibility trial (ensuring in this process that we are reaching diverse groups). To remove the chances of contamination, we will contact one smoker per household only. Where two or more smokers cohabit, the resident whose first name is alphabetically first will be selected for invitation to the study and the other resident(s) will receive the control intervention after the study has ended.

Smokers (one per household) will be sent an email and/or text or letter (dependent on GP and patient communications set up) from their GP inviting them to take part in the 'MyWay' study. This will inform them that the study will investigate the best ways of using the internet to help people to stop smoking. Where a GP practice's preferred method of contact with patients is via postal letter, 'Docmail' services will be used to distribute letters. For ethical reasons, we will not attempt to recruit people less than 16 years of age as they would typically require parental consent and parents may not be aware of their child's smoking status. The email/text/letter will include brief information and a specific web link to the study website.

\section{Recruitment via community settings}

A range of community settings have agreed to promote the study. These include libraries, leisure centres, wellbeing hubs, children's centres, the family information service and the registrar's office. A bus stop and bus-based advertising campaign will also be used to aid recruitment. We will supply each participating community setting with posters to display in prominent locations advertising the study with contact details of the research team and a community-setting specific web address to gain immediate access to the study website for participants. We will train all relevant staff in participating locations about the study to enable them to answer any basic questions and to promote it confidently. We will also offer ad hoc research assistant presence for active promotion and recruitment on site. Where staff provides a leaflet, access to the study will be either through weblink to the project website or via contact with the research team. Where a research assistant recruits on site, immediate access will be provided to the study via tablet computers. Participants will have the option to gain access to the project website without having to commit to participating.

\section{Online recruitment}

The marketing and communications team at Warwickshire County Council (WCC) are supporting all the online recruitment activities. Specifically, they are providing support for online promotion of the study via social media, targeted email marketing, Google advertising and all WCC internal and external channels, such as press releases and electronic newsletters. These ads will appear to anyone in the Warwickshire area searching for health-related products or services. We will run a 3-month long campaign to advertise the study. In addition, participating community settings will promote the study online via their social media channels.

Participants from all 3 recruitment sites will be provided with an online 'Participant Information Sheet' (PIS) prior to agreeing to take part, and have the option to gain access to the project website without having to commit to participating. 


\section{Interventions}

Please refer to figure 1 for a flow diagram illustrating participants' routes through the study. Eligible smokers will be recruited from one of three settings and provided with a link to the study webpage. This is delivered via Coventry University's secure bespoke study management software ('eNgage'). Participants will be required to endorse mandatory consent statements. Participants then complete a baseline questionnaire, which will take approximately 20 minutes to complete. Following randomisation, both groups will receive a link with nearly identical content, taking participants to either the control website or to the StopApp (intervention group). At the same time, an email containing the link will also be sent to participants to allow them to access the material at a later date. A reminder email will also be sent to all participants 2 weeks later. Participants in both conditions will be told that if they wish to book an appointment at the SSS, then they are free to do so, but in taking part are under no obligation to do so. Participants in both groups will have continued access to the content, although they have been designed to be used on a single basis. Two months after baseline data collection all participants will be asked via email to complete an online follow-up questionnaire, and invited to take part in a nested qualitative study (telephone interviews) to investigate the acceptability of the MyWay trial. The number of participants from both arms taking part in the interviews will be capped at $n=30$. Consenting participants will complete one telephone interview after follow-up. Staff from recruitment settings (community and GP) will also be invited to participate in telephone interviews about their involvement in the study. Participants will be informed that should they wish to withdraw from the study at any time they should contact the research team using the project email address (details provided on eNgage and within email communication). An email with a link to the information leaflet provided to the control group will be given to anyone who contacts us after the end of the period of recruitment to the study.

At the first appointment, stop smoking advisors will be prompted to ask participants: (1) to confirm they are taking part in the MyWay study/trial; and if yes, (2) whether they consent to the research team accessing data on their SSS use, which will be stored anonymously and (3) how they heard about the SSS. This is to ensure that consent for the access of service use data is collected by the data controller in order that they can create a report on SSS use for trial participants who consent to this. The report will identify appointments booked via StopApp and sent to a secure NHS.net email address, from which names will be cross-referenced against participant ID numbers before the data is transferred into the anonymous database maintained by the research team.

\section{Qualitative study}

At baseline, following randomisation, we will ask all participants (using a single yes/no question) if they are interested in participating in a brief follow-up telephone interview at the end of the study, regardless of whether or not they end up completing the study. For all participants that indicate an interest, we will request a contact telephone number and their consent to process this information in addition to personal data already collected. For participants providing this consent, we will select approximately 30 feasibility RCT participants and invite them to participate in the process evaluation interviews. We will apply maximum variation sampling using the demographic characteristic data collected, in order to ensure good representation of the range of consenting participants. In addition, staff contacts generated at recruitment sites during data collection will be invited to participate in process evaluation interviews. Invitation will be by telephone and those who wish to participate will be sent a follow-up email directing them to a new study page on eNgage (separate pages will be generated for staff and trial participants). On clicking 'join project', they will receive a message telling them that they will be contacted by a member of the research team to set up a mutually convenient time for the interview. The interviews will explore acceptability and user experience in line with each of the identified research objectives. They will last approximately $30 \mathrm{~min}$ and be audio recorded. Interview transcripts will be transcribed verbatim. Participants will be able to withdraw their consent for up to 2 weeks after participation in the interview, prior to data analysis. We will allow data analysis to determine saturation point and when to stop, but anticipate conducting up to 30 interviews. All qualitative data will be subject to thematic analysis $^{23}$ assisted by NVivo software.

\section{Intervention arm}

StopApp is a web-based self-administered behaviour change intervention designed to address the barriers that smokers typically face in accessing SSSs. ${ }^{1724}$ Information about users' previous quit attempts and use of SSSs is used to tailor the way in which subsequent content is framed to address any negative perceptions. Users can instantly book an appointment at a time and location of their choice, or get a reminder to reaccess the intervention at a later date. StopApp is linked to the online outcomes reporting systems owned by Pinnacle Healthcare Ltd and used by SSSs in pharmacies (PharmOutcomes) in Warwickshire (and many other local authority areas across the UK). These secure systems support service providers in recording standard SSS outcome data, such as quit dates and $\mathrm{CO}$ verified 4-week quit rates. In addition to applying coproduction and usability methods, the content of the StopApp intervention was systematically developed using the Behaviour Change Wheel approach. $^{25}$ Specific content within the app delivers 17 Behaviour Change Techniques (BCT's) identified as most useful for supporting SSS access behaviour. ${ }^{1724}$

\section{Control arm}

The control group will receive access to a webpage displaying Warwickshire's standard SSS provision 
information ('Quit4good'). The control website does not link to an online booking system and does not provide tailored routing according to the types of barriers a smoker may have to SSS access. It has not been systematically designed to address barriers to service access and is not underpinned by a theory of behaviour change or identified BCTs.

\section{Data management}

Study recruitment, consent, randomisation, provision of intervention or control content and questionnaire data collection, all take place within 'eNgage'-Coventry University's bespoke online research software hosted on their secure server. eNgage is an online research platform that includes the research pages, study information, consent and research team contact details. It is developed to work seamlessly with other web applications. On 'joining' a project, participants provide their full name and email address, on which a unique participant ID is created. Participants are then directed to Qualtrics (a separate questionnaire data inputting software) to complete the baseline, and later the follow-up measures. Participants are then directed back to eNgage where they are randomised to experimental conditions. Analytics data on participant use of the research materials (and intervention or control content) is collected by software called 'Matomo'. All data is linked by the unique participant ID.

\section{Outcomes}

\section{Baseline measures}

An online baseline questionnaire includes questions regarding demographic information (including age, gender, profession, ethnicity and postcode-for purposes of identifying indices of multiple deprivation scores); current smoking status and tobacco products used (type and quantity, including EC use); previous use of SSS; ease of internet access and motivation to quit, measured using the one-item 'Motivation to Stop Scale' (MTSS) ${ }^{26}$ and a single-item Likert scale. ${ }^{27}$ Health-related quality of life data will be collected using the EuroQOL Five Dimensions Questionnaire (EQ5D-5L) ${ }^{28}$ instrument to inform the health economic analysis ${ }^{29}$, and the ICECAP-A (ICEpop CAPability measure for Adults) ${ }^{30} 31$ instrument to measure general well-being.

\section{Two-month follow-up measures}

An online follow-up questionnaire will assess self-reports of whether participants booked an appointment and attended a SSS, set a quit date and reached a 4-week abstinence, and what prompted them to book an appointment. Pinnacle Health Ltd will run a search of the PharmOutcomes system for MyWay participants (where they consented) and provide the researchers with objective service use data. This will allow an assessment regarding the validity of sourcing objective evidence of booking, attendance (DNA rates), quit dates set and CO tested 4-week quits. We will also assess the feasibility of collecting follow-up measures of motivation using the MTSS and a single-item Likert scale ${ }^{27}$, the EQ5D-5L instrument and the ICECAP-A instrument. In addition, a bespoke resource use questionnaire will be administered, to gain insight into the costs both to individuals and to the public purse of resources accessed as a result of the use of the StopApp compared with the control.

Data on costs associated with the intervention versus usual care We will gather, through trial processes, data on the costs associated with delivering the intervention (eg, web hosting and text messages) and usual care (eg, telephone calls taken to book in appointments). We will also collect data on costs and resource use associated with promoting the intervention and usual care (eg, marketing through social media and posters on buses).

\section{Sample size}

The primary objective of this study is to assess the feasibility of recruitment to a potential future definitive RCT and therefore formal power analysis is not appropriate. However, in order to determine the target sample size, we have drawn on two sources based on the primary outcome of attendance at SSS (attend vs does not attend SSS). Researchers have recommended that external pilot and feasibility studies with binary outcome measures recruit at least 60 participants in each group (minimum $n=120$ ) and a maximum of 100 participants in each group (maximum $\mathrm{n}=200) .{ }^{32}$ In addition, our sample size calculations, based on similar definitive RCT data about recruiting smokers to trials via letters from their GPs, ${ }^{22}$ suggested that we would need to enrol 980 smokers to detect a $7 \%$ difference in attendance at SSS between control and intervention arms in a definitive trial. Based on this estimate, any trial would need to recruit 1.8 participants per day to achieve the required recruitment in 18 months. We plan to recruit for 3 months, and based on needing to recruit 1.8 participants per day, we need to reach a required sample size of 162 participants (54 per setting). With a sample size of 162 , the recruitment rate of smokers for a full RCT will be estimable with a precision (95\% CI width) of $\pm 5 \%$. We will compare recruitment rates across the three recruitment sources.

\section{Randomisation}

Sequence generation, allocation and blinding

Randomisation will be carried out through the study website during months 12-14 of the study. For this purpose, a digital bespoke randomisation algorithm (embedded within the study website) has been developed in collaboration with our statistician and the clinical trials unit at the University of Warwick. This will autorandomise at the individual level (1:1) using minimisation to ensure balance. The research team and participants will be blind to condition assignment.

\section{Statistical methods}

Feasibility and acceptability of all measures will be assessed by the level of completeness and by follow-up qualitative 
interviews with a subsample of participants. We will calculate recruitment rate via GP surgeries as a percentage of those recruited from those smokers identified on participating GPs lists. We will calculate recruitment via community and online settings as the time taken and spend required to recruit 54 participants and/or the number recruited and spend over 3 months. We will calculate the average recruitment rate per day across the three settings. We will provide percentage rates for attrition across each recruitment setting at each of baseline, intervention/ control access and 2-month follow-up.

We will look at the observed difference between intervention and control groups for bookings and attendance at SSS and use this data to support estimates for the required sample size for a definitive trial and to help to determine future trial efficacy. We will assess the level of completeness of all measures and run missing value analysis to determine whether any missing data are missing at random or whether patterns of missing data may indicate a problem with measures. We will report observed and self-reported bookings, attendances, quit dates set and 4-week quits across the intervention and control groups, and the proportion of participants agreeing to allow access to SSS data relating to smoking status and SSS attendance. We will report on numbers of higher versus lower SES status participants recruited as well as age and gender, and assess using Chi-Square $\left(\chi^{2}\right)$ analysis whether SES status, age or gender are associated with attrition at baseline, intervention/control access and 2-month follow-up.

\section{Patient and public involvement}

We have a patient and public involvement (PPI) group of 11 smokers from a range of SES backgrounds who have contributed to the design of this feasibility trial, including the participant information sheet and measures. At least one member of the PPI group will be present at study steering committee meetings throughout the duration of the feasibility trial.

\section{DISCUSSION}

The main purpose of the proposed feasibility study is to determine the necessary requirements for successful study design and data collection such that a definitive full trial can be conducted. This will largely be determined by whether one or more of the recruitment settings are able to produce the required sample size for a future main trial. We will also use data collected about SES, age and gender of those recruited and lost through attrition to understand whether SES is associated with retention in the trial. A future potential RCT will enable us to establish whether and to what extent StopApp is effective and cost-effective at increasing SSS bookings and attendance in comparison to standard methods of service promotion.

Although leaflet and letter-based methods are effective for increasing recruitment to SSS's, StopApp is unique in that its systematic evidence-based development, ${ }^{1724}$ aims to address all identified barriers to accessing SSSs while also taking account of (or will take account of during evaluation) affordability, practicability, effectiveness (and cost-effectiveness), acceptability, side effects (and safety) and equity. ${ }^{25}$ If effective and cost-effective, StopApp is instantly scalable because PharmOutcomes is already used extensively across the UK. Therefore, it would likely be delivered at even lower ongoing cost than interventions such as the cost-effective Start2quit letter and taster session, ${ }^{21}{ }^{22}$ and if well marketed could reach many more than the $5 \%-10 \%$ of smokers that services have reached so far. $^{7}$

Given that public health budgets are under increasing pressure, and evidence suggests smokers seeking help themselves are more successful at stopping than those referred by others, ${ }^{33}$ it will become increasingly important that SSSs are used by more of those making quit attempts each year to enhance their chances of success. Implementing inexpensive strategies to help this to happen will, therefore, maximise effectiveness and help to ensure optimal use of increasingly tight public health resources.

\section{Author affiliations}

${ }^{1}$ Centre for Advances in Behavioural Science, Coventry University, Coventry, UK ${ }^{2}$ Health Economics Unit, University of Birmingham, Birmingham, UK

${ }^{3}$ School of Health Sciences, University of East Anglia Faculty of Medicine and Health

Sciences, Norwich, UK

${ }^{4}$ Division of General Practice, University of Nottingham, Nottingham, UK

Contributors $\mathrm{EF}, \mathrm{KB}, \mathrm{KN}, \mathrm{KK}$ and $\mathrm{KG}$ contributed to the literature review, development of the original study protocol and drafting the initial manuscript. EF, $\mathrm{KB}, \mathrm{KN}, \mathrm{LJJ}, \mathrm{FN}$, LS and TC contributed to the trial design and outcome measures. LJJ, FN and TC offered further feasibility trial advice and contributed to the statistical and health economic analysis plan. All authors approved the final version of the manuscript.

Funding The feasibility trial is funded by the National Institute of Health Research (NIHR) Public Health Research (PHR) Programme (NIHR Portfolio number: 38004).

Competing interests None declared.

Patient consent for publication Not required.

Ethics approval Ethical approval for this study was granted by West Midlands Edgbaston Research Ethics Committee (NRES reference 18/WM/0170).

Provenance and peer review Not commissioned; peer reviewed for ethical and funding approval prior to submission.

Open access This is an open access article distributed in accordance with the Creative Commons Attribution Non Commercial (CC BY-NC 4.0) license, which permits others to distribute, remix, adapt, build upon this work non-commercially, and license their derivative works on different terms, provided the original work is properly cited, appropriate credit is given, any changes made indicated, and the use is non-commercial. See: http://creativecommons.org/licenses/by-nc/4.0/.

\section{REFERENCES}

1. ASH. Smoking statistics. 2015.

2. Ekpu VU, Brown AK. The Economic Impact of Smoking and of Reducing Smoking Prevalence: Review of Evidence. Tob Use Insights 2015;8:TUI.S15628.

3. Department of Health (DH). Local Stop Smoking Services Service Delivery and Monitoring Guidance 2011/12.

4. National Centre for Smoking Cessation \& Training (NCSCT). Why use CO-verified 4-week quit Rates as the Primary Measure of Stop Smoking Service Success? 2014.

5. West R. Stop smoking services: increased chances of quitting. NCSCT Briefing \#8. London: National Centre for Smoking Cessation and Training, 2012. 
6. HSCIC. Statistics on NHS Stop Smoking Services in England: 1 April 2013 to 31 March 2014.

7. Dobbie F, Hiscock R, Leonardi-Bee J, et al. Evaluating Long-term Outcomes of NHS Stop Smoking Services (ELONS): a prospective cohort study. Health Technol Assess 2015;19:1-156.

8. Digital NHS. Statistics on NHS Stop Smoking Services in England April 2018 to June 2018, 2018.

9. Beard E, Brose LS, Brown J, et al. How are the English Stop Smoking Services responding to growth in use of electronic cigarettes? Patient Educ Couns 2014;94:276-81.

10. Brose LS, Hitchman SC, Brown J, et al. Is the use of electronic cigarettes while smoking associated with smoking cessation attempts, cessation and reduced cigarette consumption? A survey with a 1-year follow-up. Addiction 2015;110:1160-8.

11. West R, Brown J. Trends in Electronic Cigarette use in England, 2015.

12. Roddy E, Antoniak M, Britton J, et al. Barriers and motivators to gaining access to smoking cessation services amongst deprived smokers - a qualitative study. BMC Health Serv Res 2006;6:1-7.

13. Benson FE, Stronks K, Willemsen MC, et al. Wanting to attend isn't just wanting to quit: why some disadvantaged smokers regularly attend smoking cessation behavioural therapy while others do not: a qualitative study. BMC Public Health 2014;14:695.

14. Copeland AL, Businelle MS, Stewart DW, et al. Identifying Barriers to Entering Smoking Cessation Treatment Among Socioeconomically Disadvantaged Smokers. The Journal of Smoking Cessation 2010;5:164-71.

15. Ussher M, Etter J-F, West R. Perceived barriers to and benefits of attending a stop smoking course during pregnancy. Patient Educ Couns 2006;61:467-72.

16. Vogt F, Hall S, Marteau TM. Examining why smokers do not want behavioral support with stopping smoking. Patient Educ Couns 2010;79:160-6.

17. Fulton E, Brown K, Kwah K, et al. StopApp: Using the Behaviour Change Wheel to Develop an App to Increase Uptake and Attendance at NHS Stop Smoking Services. Health Care 2016;4:31.

18. Langley T, Szatkowski L, Lewis $\mathrm{S}$, et al. The freeze on mass media campaigns in England: a natural experiment of the impact of tobacco control campaigns on quitting behaviour. Addiction 2014;109:995-1002.

19. Matcham F, McNally L, Vogt F. A pilot randomized controlled trial to increase smoking cessation by maintaining National Health Service Stop Smoking Service attendance. Br J Health Psychol 2014;19:795-809.
20. Murray RL, Coleman T, Antoniak M, et al. The effect of proactively identifying smokers and offering smoking cessation support in primary care populations: a cluster-randomized trial. Addiction 2008;103:998-1006.

21. Gilbert H, Sutton $\mathrm{S}$, Morris R, et al. Evaluating the effectiveness of using personal tailored risk information and taster sessions to increase the uptake of smoking cessation services: study protocol for a randomised controlled trial. Trials 2012;13:195.

22. Gilbert H, Sutton S, Morris R, et al. Effectiveness of personalised risk information and taster sessions to increase the uptake of smoking cessation services (Start2quit): a randomised controlled trial. The Lancet 2017;389:823-33.

23. Braun V, Clarke V. Using thematic analysis in psychology. Qual Res Psychol 2006;3:77-101.

24. Fulton E, Kwah K, Wild S, et al. Lost in Translation: Transforming Behaviour Change Techniques into Engaging Digital Content and Design for the StopApp. Health Care 2018;6:75.

25. Michie S, Atkins L, West R. The behaviour change wheel: a guide to designing interventions. Great Britain: Silverback Publishing, 2014.

26. Kotz D, Brown J, West R. Predictive validity of the Motivation To Stop Scale (MTSS): A single-item measure of motivation to stop smoking. Drug Alcohol Depend 2013;128:15-19.

27. Hummel K, Candel MJJM, Nagelhout GE, et al. Construct and Predictive Validity of Three Measures of Intention to Quit Smoking: Findings From the International Tobacco Control (ITC) Netherlands Survey. Nicotine \& Tobacco Research 2018;20:1101-8.

28. The EuroQol Group. EuroQol - a new facility for the measurement of health-related quality of life. Health Policy 1990;16:199-208.

29. Devlin N, Shah K, Feng Y, et al. Valuing Health-Related Quality of Life: An EQ-5D-5L Value Set for England, 2016.

30. Al-Janabi H, N Flynn T, Coast J. Development of a self-report measure of capability wellbeing for adults: the ICECAP-A. Quality of Life Research 2012;21:167-76.

31. Al-Janabi H, Peters TJ, Brazier J, et al. An investigation of the construct validity of the ICECAP-A capability measure. Quality of Life Research 2013;22:1831-40.

32. Teare MD, Dimairo M, Shephard N, et al. Sample size requirements to estimate key design parameters from external pilot randomised controlled trials: a simulation study. Trials 2014;15:264.

33. Borland R, Partos TR, Yong H-H, et al. How much unsuccessful quitting activity is going on among adult smokers? Data from the International Tobacco Control Four Country cohort survey. Addiction 2012;107:673-82. 